\title{
Probing the spectral and temporal structures of high-order harmonic generation in intense laser pulses
}

\author{
Xiao-Min Tong* and Shih-I Chu \\ Department of Chemistry, University of Kansas, and Kansas Center for Advanced Scientific Computing, Lawrence, Kansas 66045
}

(Received 16 July 1999; published 5 January 2000)

\begin{abstract}
We present an $a b$ initio three-dimensional quantum study of high-order harmonic generation (HHG) of atomic $\mathrm{H}$ in intense pulsed laser fields. Accurate time-dependent wave functions are obtained by means of the time-dependent generalized pseudospectral method recently developed and wavelet transform is used to perform time-frequency analysis of the resulting HHG power spectra. The results reveal striking details of the spectral and temporal fine structures of HHG, providing insights regarding HHG mechanisms in different energy regimes and benchmark data for testing the validity of existing HHG models.
\end{abstract}

PACS number(s): 42.65.Ky, 32.80.Wr, 42.50.Hz

Recently a great deal of attention has been devoted to the study of multiple high-order harmonic generation (HHG) processes in intense short laser pulses [1-3]. Besides its fundamental interest for strong-field atomic and molecular physics, the HHG provides a potential tunable coherent light source in the extreme ultraviolet (XUV) region, a so-called " "tabletop synchrotron" [2]. Further, the HHG may lead to a promising way for generating subfemtosecond (attosecond) pulses of radiation of high frequency $[4,5]$. For optimal control of the HHG processes, it is essential to have a thorough understanding of the spectral and temporal structures of the high-order harmonics and the detailed underlying mechanisms for HHG. The first experimental observation of the temporal coherence of HHG has recently been reported [6]. In this Rapid Communication, we present an ab initio threedimensional (3D) precision quantum calculation of the timedependent Schrödinger equation of atomic $\mathrm{H}$ in intense laser pulses and perform a detailed wavelet time-frequency analysis of the spectral and temporal structures of HHG. The results reveal rich information on the fine structures of timefrequency spectra, providing insights regarding the underlying mechanisms for HHG. Further, the present study provides a benchmark calculation that can be used for the examination of the range of validity of the existing models for HHG [7-13].

In the presence of linearly polarized (LP) laser fields, most HHG spectra show a similar generic behavior: a sharp decline of the harmonic intensity for the first few harmonics, then a plateau consisting of many harmonics, followed by a cutoff. A conceptual understanding of the origin of the harmonics with energies much in excess of the ionization potential $I_{p}$ is provided by the three-step quasiclassical model of Corkum [7] and Kulander et al. [8]. The mechanisms for the generation of lower and threshold harmonics are less well understood. The quasiclassical model involves tunnel ionization, acceleration of the free electron in the monochromatic laser field, and recollision of the electronic wave packet with the parent ionic core. Based on this model, the cutoff energy is predicted to be $E_{c}=I_{p}+3.17 U_{p}$, where $U_{p}$ is the ponderomotive energy $[7,8]$. This cutoff law was confirmed by

\footnotetext{
*Present address: Cold trapped ions, JST International Project, Axis Chofu 3F, 1-40-2 Fuda Chofu, Tokyo 182-0024, Japan.
}

Lewenstein et al. [9], using a quantum treatment of model systems in the strong-field approximation. Later, Kan et al. [10] extended the Lewenstein model to study the HHG features in pulsed fields. More recently, Protopapas et al. [11] presented a bremsstrahlung model for harmonic emission. Finally, Watson et al. [12] extended the Protopapas model to explain the origin of blue shift but no discussion of the fine structure of the HHG peaks was given. In this Rapid Communication, we present a complete analysis of the underlying mechanisms for the production of harmonics in different intensity and energy regimes based on accurate $a b$ initio $3 \mathrm{D}$ quantum calculations.

We consider the solution of the time-dependent Schrödinger equation of atomic $\mathrm{H}$ in LP pulsed laser fields, in atomic units: $i \partial / \partial t \quad \psi(\mathbf{r}, t)=H(t) \psi(\mathbf{r}, t)$, where $H(t)=H_{0}$ $+V(t)$. Here $H_{0}$ is the unperturbed atomic Hamiltonian and $V(t)$ is the coupling of the electron with the laser pulse: $V(t)=-z E f(t) \sin \left(\omega_{0} t\right)$, where $E$ is the peak amplitude and $f(t)$ the pulse shape ( $\sin ^{2}$ pulse is used). The propagation of the wave function is performed by means of the recently developed time-dependent generalized pseudospectral (TDGPS) method [14]. The radial coordinate $r$ is discretized by the generalized pseudospectral technique [15], allowing nonuniform spatial grid spacing: a denser mesh near the origin and a sparser mesh for the outer regime. This nonuniform discretization is essential, since it allows accurate treatment of the physically most significant short-range regime as well as the use of a considerably smaller number of grid points. The time propagation of the wave function is achieved by the second-order split-operator method in the energy representation $[14,16]$

$$
\begin{aligned}
\psi(\mathbf{r}, t+\Delta t) \simeq & e^{-i \hat{H}_{0} \Delta t / 2} e^{-i \hat{V}(r, \theta, t+\Delta t / 2) \Delta t} e^{-i \hat{H}_{o} \Delta t / 2} \psi(\mathbf{r}, t) \\
& +O\left(\Delta t^{3}\right) .
\end{aligned}
$$

As demonstrated in our recent studies in both strong-field HHG processes $[14,16]$ and Rydberg-atom high-resolution spectroscopy [17], the TDGPS procedure is computationally more efficient and accurate than the conventional timedependent techniques using equal-spacing grid discretization. A high-accuracy time-dependent wave function is essential to the present study of the spectral and temporal structures of HHG. The unitarity of the wave function is automatically preserved by Eq. (2), and the norm of the 


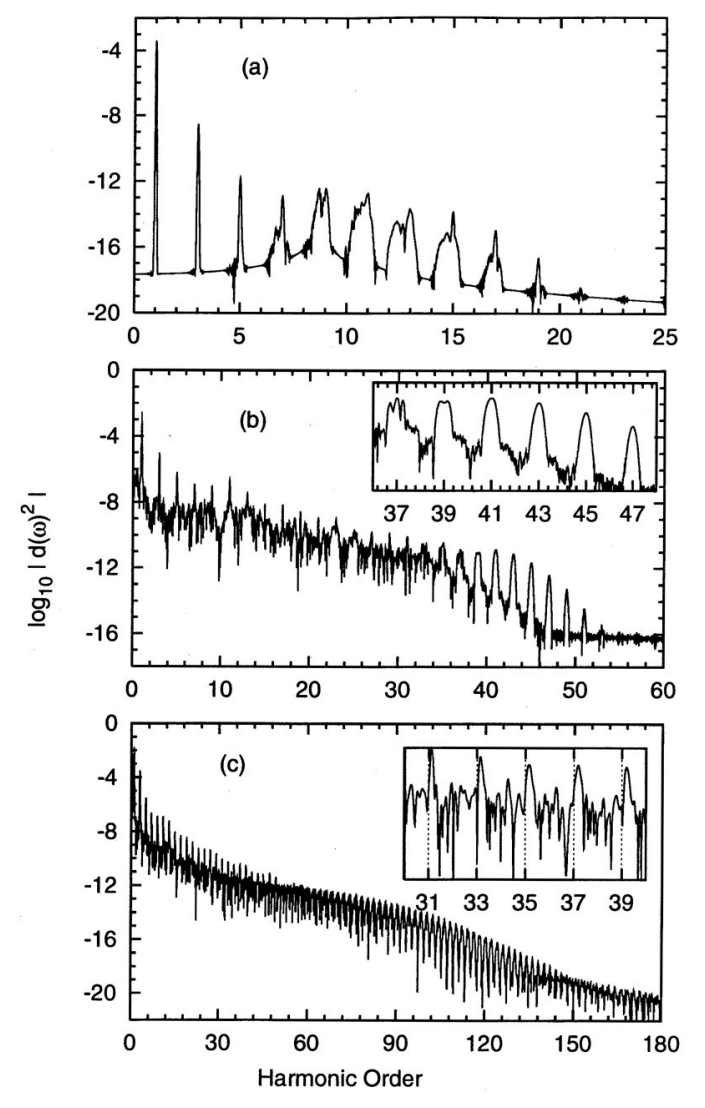

FIG. 1. The power spectrum of HHG of atomic $\mathrm{H}$ in the pulsed laser fields with laser peak intensity (a) $I=10^{13} \mathrm{~W} / \mathrm{cm}^{2}$, (b) $I=10^{14} \mathrm{~W} / \mathrm{cm}^{2}$, and (c) $I=5 \times 10^{14} \mathrm{~W} / \mathrm{cm}^{2}$. The laser pulse has a $\sin ^{2}$ pulse shape with a duration of 60 o.c. The laser wavelength used is $1064 \mathrm{~nm}$. Shown in the insets are some spectral fine structures.

field-free wave function is preserved to at least ten digits of accuracy during the whole time propagation. The quality of the time-dependent wave function obtained by the TDGPS procedure is further demonstrated by the excellent agreement of the HHG power spectra in the length and acceleration forms (from the lowest harmonic to the cutoff) [14].

The laser parameters used in the present study are $\lambda$ $=1064 \mathrm{~nm}$ and laser intensities $I=10^{13}, 10^{14}$, and 5 $\times 10^{14} \mathrm{~W} / \mathrm{cm}^{2}$, corresponding to the Keldysh parameter [18] $\gamma=\sqrt{I_{p} /\left(2 U_{p}\right)}$, equal to $2.50,0.80$, and 0.36 , respectively. The time-dependent-induced dipole moment and dipole acceleration are calculated by the use of the time-dependent wave function obtained by the TDGPS method. In the present calculation, the unperturbed ground-state energy of atomic $\mathrm{H}$ is at least of 12 digits of accuracy. In the time propagation, the radial grid extends to 100 a.u., and a soft adsorber [14] is placed at 60 a.u. The time step used is 0.15 a.u. Up to 400 radial grid points (for each partial wave) and 50 partial waves are used to achieve full convergence of the time-dependent wave functions and HHG power spectra. Figures 1(a)-1(c) show the HHG power spectra for the three intensities indicated above with a laser pulse length of 60 optical cycles (o.c.).

The detailed spectral features are significantly different for the three cases. For the lower intensity case [Fig. 1(a)] dominated by the multiphoton mechanism, the most salient feature is the several broad peaks with fine structure (due mainly to the effects of excited states) around the ionization threshold. As the laser intensity is increased, the influence of the excited states becomes less important, as seen in Figs. 1(b) and 1(c). A prominent feature of the intermediate intensity case [see the inset in Fig. 1(b)] is the apparently broad but structureless harmonic peaks near the cutoff. However, the harmonics in the midplateau regime (39th harmonic and below) show peak splitting. For the strongest intensity case considered here, the most noticeable spectral features are the blue shift and the peak splitting [see the inset in Fig. 1(c)]. These features can be seen throughout the plateau harmonics and become increasingly more prominent as the harmonic order is increased.

To explore the detailed spectral and temporal structures of HHG, we perform the time-frequency analysis by means of the wavelet transform $[19,20]$ of the induced dipole (or dipole acceleration),

$$
A_{w}\left(t_{0}, \omega\right)=\int d(t) w_{t_{0}, \omega}(t) d t \equiv d_{\omega}(t),
$$

with the wavelet kernel $w_{t_{0}, \omega}(t)=\sqrt{\omega} W\left(\omega\left(t-t_{0}\right)\right)$. For the harmonic emission, a natural choice of the mother wavelet is given by the Morlet wavelet $[19,20]$

$$
W(x)=(1 / \sqrt{\tau}) e^{i x} e^{-x^{2} / 2 \tau^{2}} .
$$

We have tested the dependence of $d_{\omega}(t)$ on the parameter $\tau$ by varying its value from 5 to 30 . Although the absolute value of $d_{\omega}(t)$ changes a little, the general pattern does not change. In the discussion below we choose $\tau=15$ to perform the wavelet transform.

Figures 2(a)-2(c) show the 3D graphs of the modulus of the time-frequency profiles corresponding to the three laser intensities $I$ indicated in Figs. 1(a)-1(c), revealing striking and vivid details of the spectral and temporal structures. (For better graphic resolution within the limited space, we choose to present the data for the shorter laser pulse case with 15 optical cycles. The time-frequency profiles are qualitatively similar for the cases of 15 and 60 optical cycles.) Several salient features are noticed. First, for the lowest few harmonics, the time profile (at a given frequency) shows a smooth function of the driving laser pulse. This is an evidence that the multiphoton mechanism dominates this lower-energy regime: the probability of absorbing $N$ photons is roughly proportional to $I^{N}$ and $I$ is proportional to $E^{2}(t)$. As the harmonic order is increased and closes to the ionization threshold, the time profiles [see, for example, the ninth harmonic in Fig. 2(a)] develop extended structures. This may be attributed to the near resonant effect of the excited states (particularly the $2 p$ state). Second, for the two higher laser intensity cases, Figs. 2(b) -2 (c), the most prominent feature is the development of fast burst time profiles for those harmonics well above the ionization threshold, indicating that the tunneling mechanism is at work here. Moreover, for each burst, we see that the electron emits a continuous frequency profile. This is clear evidence of the existence of the bremsstrahlung radiation emitted by each recollision of the electron wave packet with the parent ionic core. In contrast, the 

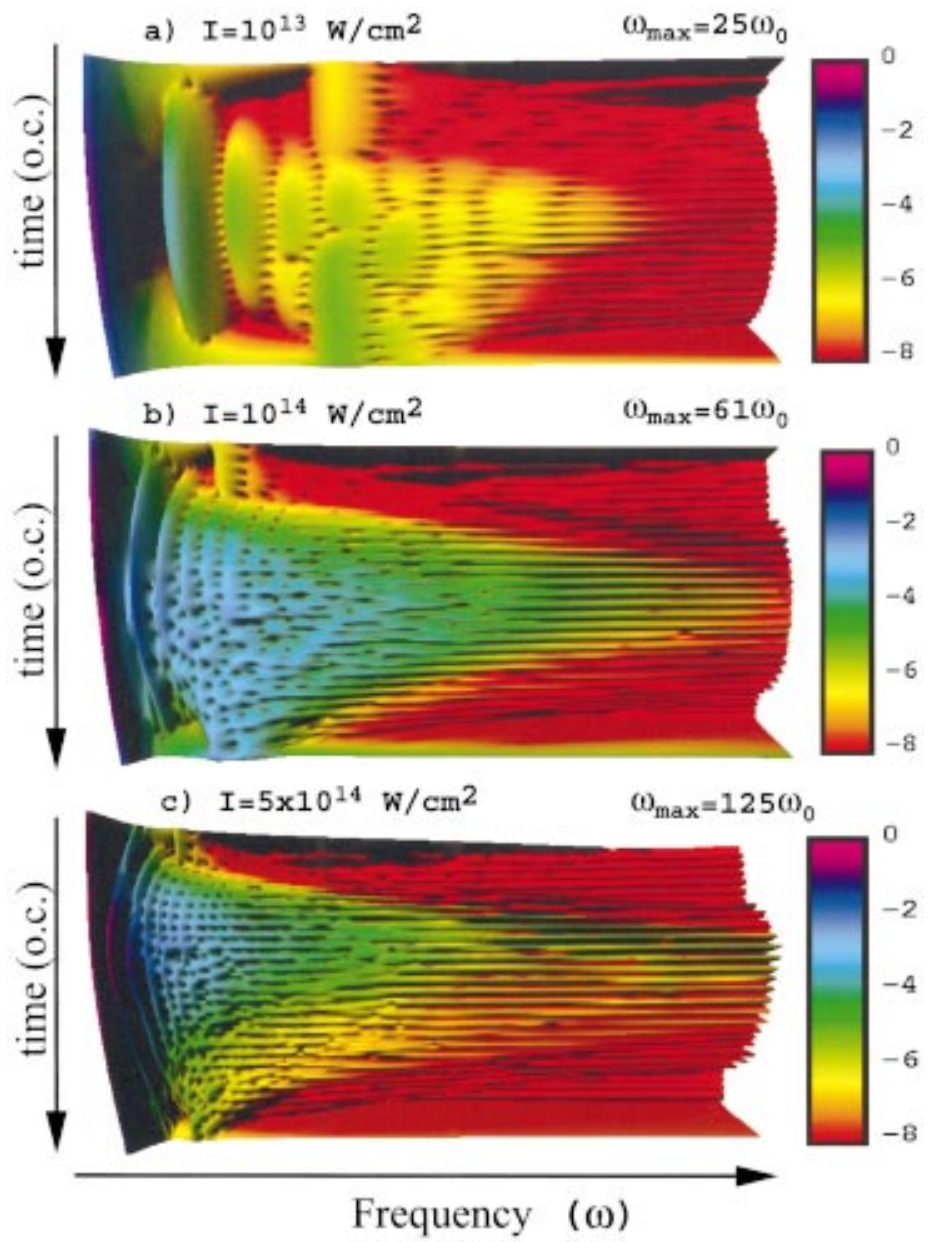

FIG. 2. (Color) The 3D time-frequency spectra (modulus) of atomic $\mathrm{H}$ in pulsed laser fields. The laser parameters are the same as those in Fig. 1 except the pulse duration is 15 optical cycles instead. The colors shown are in logrithmic scale (in powers of 10). (multiphoton-dominant) lowest-order harmonics form a continuous time profile at a given frequency. In the intermediate energy regime where both multiphoton and tunneling mechanisms prevail, the time-frequency profiles show a netlike structure, as seen in Figs. 2(b) and 2(c). To our knowledge, this is the first presentation of HHG time-frequency profiles that exhibit the subtle fine details of spectral and temporal structures for the whole energy range.

We now discuss the origin of the power spectrum pattern near the cutoff in Fig. 1(b). Figures 3(a) and 3(b) show, respectively, the time profiles of the 45 th and 37 th harmonics obtained by the cross section of the 3D time-frequency profile [similar to that in Fig. 2(b)] at a given harmonic frequency for the case of laser pulse length of 60 o.c. Figure 3 (a) depicts a representative time profile near the cutoff that exhibits two bursts within each optical cycle. Each burst is due to the recollision of the electronic wave packet with the ionic core. Also shown in Fig. 3(a) (vertical dashed lines) are the electron returning times (with phase $\omega t=342^{\circ}$ or $162^{\circ}$ ) predicted by the quasiclassical model $[7,8,21]$. The agreement of the full ( $a b$ initio) quantum and quasiclassical prediction on the electron-ion core recollision times is rather striking, indicating the validity of the quasiclassical model in this tunneling limit. Moreover, as the electron returning energy is below the cutoff, there exists two returning trajectories according to the semiclassical model and stationary phase analysis of the dipole acceleration $[9,10]$ : the first ("short") trajectory returns at a phase $(\omega t)$ less than $342^{\circ}$ (or $162^{\circ}$ ), and the second ("long") trajectory at a phase larger than $342^{\circ}$ (or $162^{\circ}$ ). This is also confirmed in Fig. $3(\mathrm{~b})$, where we show a representative time profile for harmonics below the cutoff.

Also shown in Figs. 3(a)-3(b) are the "dynamical phases" $\theta\left(t_{q}\right)$ (denoted by black dots) obtained from the wavelet transform of the induced dipole (or dipole acceleration), corresponding to each instant $\left(t_{q}\right)$ of electron-ion core recollision: $\quad d_{\omega}\left(t_{q}\right)=\left|d_{\omega}\left(t_{q}\right)\right| e^{-i S\left(t_{q}\right)}$, where $S\left(t_{q}\right)=\omega t_{q}$ $+\theta\left(t_{q}\right)$. The dynamical phases of the cutoff harmonics [Fig. 3(a)] show a time profile mimicking the laser pulse shape. For harmonics below the cutoff [Fig. 3(b)], it is seen that the "long" trajectory has stronger phase dependence on laser intensity than that of the "short" one, an observation that was also inferred by previous model calculations [10]. We note that the instantaneous frequency $\tilde{\omega}(t)$ can be obtained by $\tilde{\omega}(t)=d S(t) / d t=\omega+d \theta(t) / d t$. When $d \theta(t) / d t>0$, corresponding to the rising part of the laser pulse, a blue shift of the spectrum will occur. Conversely, a red shift will appear when $d \theta(t) / d t<0$, corresponding to the falling part of the laser pulse. The overall frequency shift of the harmonic peak depends on the relative contribution of the rising and falling parts of the laser pulse. For low to intermediate laser intensities, the two contributions cancel exactly and there is no net frequency shift. For intense laser pulses, when the electron is ionized before the peak intensity is reached, the rising part of 


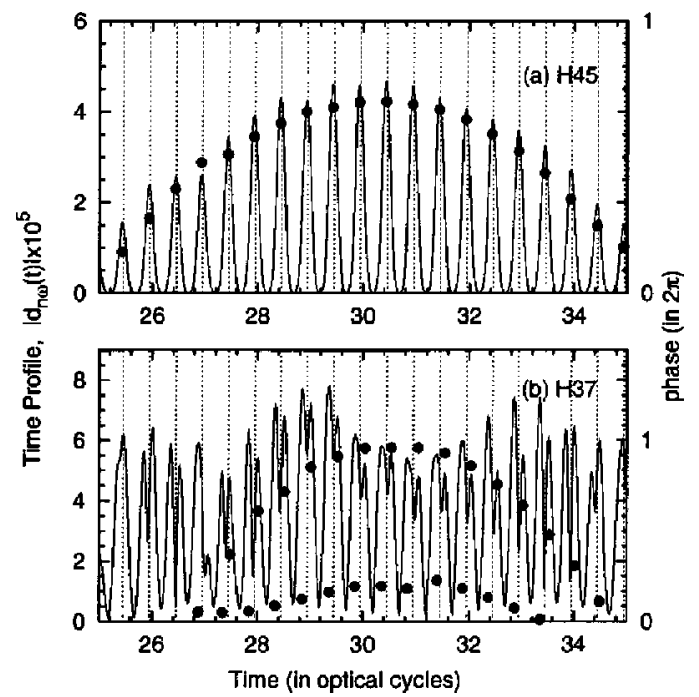

FIG. 3. The time profiles of the 45th (near the cutoff) and 37th (below the cutoff) harmonics for atomic $\mathrm{H}$ in pulsed laser fields. The laser parameters are the same of those in Fig. 1(b). The vertical dashed lines are the electron returning times predicted by the quasiclassical model.

the pulse dominates the process, and a net blue shift will occur.

To explore the origin of the peak splitting of harmonics in the plateau regime (dominated by the tunneling mechanism) as seen in Figs. 1(b) and 1(c), Fig. 4 shows the time profile of a representative (93rd) harmonic corresponding to the highest intensity case [Fig. 1(c)]. Significant ionization occurs in this intensity, and the time profiles indicate that the harmonic emission occurs before the peak intensity of the laser pulse is reached. Further, the deviation of quantum electron returning times from the quasiclassical prediction (vertical dashed lines) increases with increasing laser pulse intensity. The 93rd harmonic (shown in the inset) shows a significant blue shift and a three-peak fine structure. Figure 4 shows the time profiles at three arbitrary frequency positions (denoted by 1, 2, and 3) within the 93rd harmonic. Strikingly, their time profiles nearly coincide. This indicates that

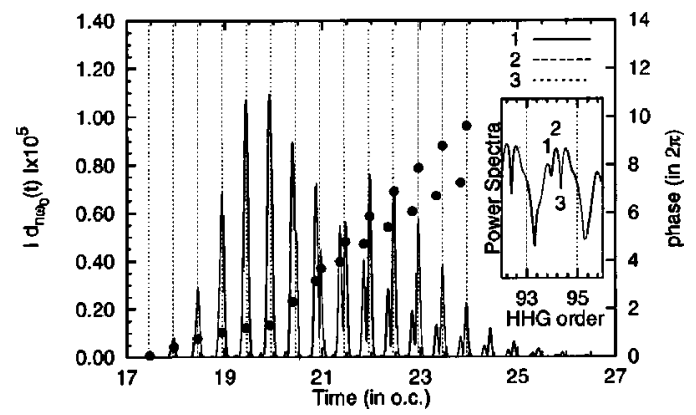

FIG. 4. The time profiles of the subpeaks of the 93rd harmonic of atomic $\mathrm{H}$ in intense pulsed laser fields. The laser parameters are the same as those in Fig. 1(c).

all the harmonic subpeaks within a given plateau harmonic are produced by the same mechanism, namely, they are produced by the interference in time of all the bremsstrahlung radiation emitted from all the returning electronic wave packets within the incident laser pulse duration. Our ab initio results thus confirm the prediction of the Protopapas model [11] for the harmonic emission. To our knowledge, this is the first $a b$ initio calculation exhibiting the details of the time profiles of the subpeak harmonics. Also shown in Fig. 4 are the dynamical phases $\theta\left(t_{q}\right)$ obtained from the wavelet analysis. In this high-intensity case, we see that both the "short" and "long" returning electronic wave packets now exhibit a sensitive dependence on laser intensity, although the "long" trajectory still shows stronger intensity dependence, as seen in the lower intensity cases.

In conclusion, we have presented in this Rapid Communication a completely $a b$ initio precision 3D calculation of the fine structure of the spectral and temporal profiles of HHG in different intensities and energy regimes. Our analysis provides insights into the detailed HHG mechanisms and a benchmark calculation for the examination of the validity of various existing HHG models.

This work was partially supported by the NSF. The Origin2000 supercomputer time was supported by the Kansas Center for Advanced Scientific Computing.
[1] A. L'Huillier et al., in Atoms in Intense Laser Fields, special issue of Adv. At., Mol., Opt. Phys. Suppl. 1, 139 (1992), edited by M. Gavrila.

[2] Z. Chang et al., Phys. Rev. Lett. 79, 2967 (1997); Ch. Spielmann et al., Science 278, 661 (1997); M. Schnürer et al., Phys. Rev. Lett. 80, 3236 (1998).

[3] For theoretical investigation of HHG spectra based on a 3D numerical solution of Schrödinger equation, see, for example, J.L. Krause et al., Phys. Rev. Lett. 68, 3535 (1992); A. de Bohan et al., ibid. 81, 1837 (1998).

[4] I.P. Christov et al., Phys. Rev. Lett. 78, 1251 (1997).

[5] P. Antoine et al., Phys. Rev. A 56, 4960 (1997).

[6] M. Bellini et al., Phys. Rev. Lett. 81, 297 (1998).

[7] P.B. Corkum, Phys. Rev. Lett. 71, 1994 (1993).

[8] K. C. Kulander et al., in Super-Intense Laser-Atom Physics, Vol. 316 of NATO Advanced Study Institute Series B: Physics, edited by P. B. Piraux et al. (Plenum, New York, 1993).
[9] M. Lewenstein et al., Phys. Rev. A 49, 2117 (1994).

[10] C. Kan et al., Phys. Rev. A 52, R4336 (1995).

[11] M. Protopapas et al., Phys. Rev. A 53, R2933 (1996).

[12] J.B. Watson et al., Phys. Rev. A 55, 1224 (1997).

[13] P. Antoine et al., Laser Phys. 7, 594 (1997).

[14] X.M. Tong and S. I. Chu, Chem. Phys. 217, 119 (1997).

[15] G. Yao, and S. I. Chu, Chem. Phys. Lett. 204, 381 (1993); J. Wang, et al., Phys. Rev. A 50, 3208 (1994).

[16] X.M. Tong, and S. I. Chu, Phys. Rev. A 57, 452 (1998).

[17] S.I. Chu et al., Chem. Phys. Lett. 294, 31 (1998).

[18] L.V. Keldysh, Zh. Éksp. Teor. Fiz. 47, 1945 (1964) [Sov. Phys. JETP 20, 1307 (1965)].

[19] C. K. Chui, An Introduction to Wavelets (Academic Press, New York, 1992).

[20] P. Antoine et al., Phys. Rev. A 51, R1750 (1995); M. Gaarde et al., ibid. 57, 4553 (1998).

[21] S.C. Rae et al., Phys. Rev. A 50, 3438 (1994). 